THE QUALITATIVE METHODOLOGY FOR THE STUDY OF ENVIRONMENTAL PROBLEMS AS A PEDAGOGICAL PRACTICE IN BIOLOGY

A METODOLOGIA QUALITATIVA PARA O ESTUDO DE PROBLEMAS AMBIENTAIS COMO PRÁTICA PEDAGÓGICA EM BIOLOGIA

Carelia Rayen Hidalgo

D https://orcid.org/oooo-0oo2-4531-6987

Iris Delfin

https://orcid.org/oooo-0oo2-1763-5315

\begin{abstract}
REVISTA PEDAGÓGICA
Revista do Programa de Pós-graduação em Educação da Unochapecó | ISSN 1984-1566 Universidade Comunitária da Região de Chapecó | Chapecó-SC, Brasil Como referenciar este artigo: HIDALGO, C. RAYEN; DELFIN, I. La metodología cualitativa para el estudio de problemáticas ambientales como práctica pedagógica en biología. Revista Pedagógica, Chapecó, v. 21, p. 557-576, 2019.

DOI: http://dx.doi.org/10.22196/rp.v22io.5019
\end{abstract}

RESUMEN: La dimensión ambiental en la praxis educativa requiere de la permanente innovación que promueva la contextualización de la enseñanza. En tal sentido, la investigación tuvo como propósito valorar la Práctica Pedagógica (PP) de biología de educación media general en el Liceo Dr. Carlos Emilio Muñoz Oraá, Venezuela. El artículo se presenta como un análisis descriptivo del cuestionamiento de una PP usando la investigación acción para el aprendizaje de problemáticas ambientales con la Metodología Cualitativa (MC). La docente mantuvo un proceso crítico y reflexivo sobre la PP con 75 estudiantes de tres secciones de cuarto año. Fundamentado en diagnósticos se diseñó, ejecutó y evaluó un plan de trabajo coherente a los objetivos académicos de la asignatura. Se logró la aplicación y cognición sobre la MC por parte de los estudiantes en un trabajo contextualizado sobre problemáticas ambientales de sus comunidades. También el aprendizaje significativo fue sistematizado en proyectos de investigación de los estudiantes. Se logró un impacto en la dimensión social, ambiental y pedagógica, al cumplir principios y objetivos de la educación ambiental y el currículo.

Palabras clave: metodología cualitativa, problemáticas ambientales, práctica pedagógica, enseñanza de biología.

RESUMO: A dimensão ambiental na prática educacional requer inovação permanente que promova a contextualização da educação. Nesse sentido, o objetivo da pesquisa foi avaliar a Prática Pedagógica (PP) do ensino de biologia na Escola Secundária Dr. Carlos Emilio Muñoz Oraá, Venezuela. O artigo é apresentado como uma análise descritiva do questionamento de um PP utilizando pesquisa-ação para a aprendizagem de problemas ambientais com a Metodologia Qualitativa (MQ). A professora manteve um processo crítico e reflexivo sobre o PP com 75 alunos de três secções do quarto ano ensino meio. Com base nos diagnósticos, foi elaborado, executado e avaliado um plano de trabalho coerente com os objetivos acadêmicos do currículo. A aplicação e cognição em MQ pelos alunos foram alcançadas em um trabalho contextualizado sobre os problemas ambientais de suas comunidades. Também aprendizado significativo foi sistematizado em projetos de pesquisa de estudantes. Impacto foi alcançado na dimensão social, ambiental e pedagógica, cumprindo os princípios e objetivos da educação ambiental e do currículo.

Palavras-chave: metodologia qualitativa, problemas ambientais, prática pedagógica, ensino de biologia.

ABSTRACT: The environmental dimension in educational praxis requires permanent innovation that promotes the contextualization of education. In this regard, the purpose of the research was to assess the Pedagogical Practice (PP) at biology at the Dr. Carlos Emilio Muñoz Oraá High School, Venezuela. The article is presented as a descriptive analysis of the questioning of a PP using action research for the learning of environmental problems with the Qualitative Methodology (QM). The teacher maintained a critical and reflective process about the PP with 75 students from three fourth-year sections of general secondary. Based on diagnoses, a work plan coherent to the academic objectives of the subject was designed, executed and evaluated. The application and cognition on QM by students was achieved in a contextualized work on environmental problems of their communities. Also significant learning was systematized in student research projects. An impact was achieved in the social, environmental and pedagogical dimension, by complying with the principles and objectives of environmental education and the curriculum.

Keywords: qualitative methodology, environmental problems, pedagogical practice, biology teaching. 
Amenizar la enseñanza con la hermosa palabra, con la anécdota oportuna, y la relación de cada conocimiento con la vida. (Pensamientos pedagógicos de la poetisa chilena, Gabriela Mistral 1889-1957)

\section{Introducción}

La calidad de la educación es inherente al quehacer educativo y es consecuencia de la acción transformadora del docente y del estudiante en las aulas. El contexto actual requiere que las instituciones educativas transformen su modelo de enseñanza centrado en la repetición de cosas sabidas, reproducción y consumo de información. En tanto se espera que el estudiante sea sujeto activo y responsable de la construcción de nuevos saberes a través de su aprendizaje, entre dichos saberes el ambiental.

Ante tales demandas se requiere que el docente sea un investigador y el aula un espacio de investigación, diálogo y desarrollo profesional, donde se cuestionan los conocimientos y Práctica Pedagógica (PP). Entonces el docente necesita mantener comprensión, reflexión e interpretación de la realidad para la creación de nuevas situaciones, a partir de los problemas de la PP cotidiana, con la finalidad de mejorarla o transformarla. Entonces el docente integra en su práctica la función investigadora como medio de autodesarrollo profesional y oportunidad para mejorar la calidad de la educación.

Se entiende entonces, que la investigación basada en la transformación ambiental y educativa debe plantearse en la realidad escolar y comunitaria, así contar con los aportes que puedan brindar los docentes desde sus PP. Dadas estas consideraciones, se requiere de un proceso educativo pertinente con la realidad, relacionado con lo que ocurre en el entorno, tal como lo plantea la educación ambiental, la contextualización.

Atendiendo a este discurso, durante tres períodos escolares de PP, dentro del Liceo "Dr. Carlos Emilio Muñoz Oráa", fue llevada a cabo la investigación de problemáticas ambientales poniendo en práctica los pasos del método científico, a través de un contenido de aprendizaje denominado "Introducción a la Metodología de la Investigación”, impartida en la asignatura Biología, dentro de cuarto año del nivel media general. Sin embargo la reflexión sobre dicha PP fue que la misma estuvo limitada a un método fundamentado en la revisión bibliográfica, la aplicación de encuestas y el análisis estadístico. En la PP no se generaron reflexiones profundas ni críticas, por parte de los estudiantes, sobre la realidad ambiental, ni impactos positivos sobre ella. Culminaba siendo una práctica de aula común, que no rompe con los viejos paradigmas de enseñanza-aprendizaje.
* Profesora de Ecología y Elementos del Ecosistema (1998-2018) y Profesora de la electiva Educación Ambiental en el manejo de Cuencas Hidrográficas, (desde 2000 al 2007) en el Decanato de Agronomía de la UCLA del Decanato de Agronomía de la Universidad Centroccidental Lisandro Alvarado, en categoría de Titular (desde 2017) con dedicación TC; coordinadora de la Comisión de Ambiente de la UCLA (20072018); docente en Educación Ambiental (CUFT, desde 1994 al 2015); fundadora y creadora (1999) y miembro activo hasta el año 2018 del "Centro Demostrativo de Tecnologías Agroecológicas para el Semiárido" (CenecoAgro). Promotora en la creación y coordinadora de la Red Venezolana de Universidades por el Ambiente (REDVUA) (2013-2018). Creadora y facilitadora (2012-2018) de la Unidad Curricular de Educación Ambiental para la Sostenibilidad, en el Programa de Formación y Actualización Docente de la UCLA. Docente invitada en maestrías y doctorados en la UPEL (IPC e IPB) y UNELLEZ, en las sedes Guanare, Acarigua, Apure y Cojedes. Docente invitada en cursos de educación ambiental en la Universidad Peruana Cayetano Heredia. Invitada en actividades de desarrollo rural, agroecología, gestión y educación ambiental en diversas instituciones públicas y privadas para dictar cursos, talleres o conferencias. Profesora Visitante Extranjera en el Programa de Postgrado en Educación Ambiental (PPGEA) en la Universidad Federal de Rio Grande, Brasil (desde octubre 2018).

E-mail: careliahidalgo@gmail.com

** E-mail: irisdegonzalez2011@hotmail. com 
Sin embargo, los nuevos métodos requieren que el estudiante se enfrente a una realidad y pueda reflexionar sobre ella, que conozca los fenómenos sociales, educativos, culturales y ambientales del contexto donde hace vida social, ciudadana. Era necesario pensar en una PP, donde se forme un ciudadano con una nueva comprensión de la relación hombre-naturaleza-sociedad. En este sentido la educación ambiental es uno de los encargos sociales en la educación venezolana, de allí la trascendencia de diseñar un modelo pedagógico que logrará abordar la complejidad ambiental y coadyuve con el logro de ese ciudadano integral.

Por la confrontación de la experiencia y demandas educativas, se consideró pertinente incorporar, en el contenido, la investigación cualitativa, que les permitiera a los estudiantes observar, reflexionar y generar propuestas de cambio en función de las problemáticas ambientales de sus realidades. Para ello, se pusieron en práctica estrategias didácticas para la inserción de la metodología cualitativa así como herramienta de validación del impacto pedagógico, social y ambiental del quehacer educativo. La investigación se logró por la docente y 75 estudiantes correspondientes a tres secciones de biología en cuarto año de educación media general, del Liceo "Dr. Carlos Emilio Muñoz Oráa" ubicado en el municipio Guanare, estado Portuguesa. Sin ser los jóvenes los actores esenciales de la investigación, fueron actores fundamentales de la PP.

En tal sentido surgieron las siguientes preguntas ¿Cómo será la práctica pedagógica de biología, en educación media general, que permita el análisis de problemáticas ambientales desde la enseñanza de la metodología cualitativa?¿Cuál será el impacto generado por la inclusión de la metodología cualitativa aplicada por estudiantes del nivel media general para el abordaje de problemas ambientales desde la asignatura biología? Así la presente investigación tuvo como propósito: Valorar la práctica pedagógica con la inserción de la metodología cualitativa para el estudio de problemáticas ambientales en cuarto año de biología del Liceo Dr. Carlos Emilio Muñoz Oraá.

La PP se justificó en el Currículo Nacional Bolivariano $(\mathrm{CNB}, 2007)$ que incluye atender a jóvenes entre los doce (12) y diecinueve (19) años de edad, con la finalidad de "lograr la formación integral de los y las adolescentes y jóvenes, atendiendo a los fines y principios que inspiran la República”. Este currículo pretende promover el pensamiento crítico y reflexivo, que permita el análisis de la realidad para transformarla desde una nueva conciencia crítica. Entre otras cosas, propicia una actitud responsable y protagónica en relación con la preservación y conservación del ambiente y la salud, vinculándose a su comunidad en la búsqueda de soluciones a los problemas ambientales. Entre los ejes integradores, pilares, componentes y áreas de aprendizaje, se incluye el ser humano y su interacción con otros componentes del ambiente. En la asignatura biología se incorporan los contenidos de aprendizaje: cultura 
ambiental responsable en la ejecución de actividades para la protección del ambiente y metodología de investigación.

\title{
Bases conceptuales
}

\section{Práctica pedagógica como oportunidad de espacio investigativo}

Esta investigación se sustentó en que la Práctica Pedagógica (PP) está continuamente en un proceso de transformación. Al respecto Rodríguez (2006), señala que

\begin{abstract}
"la práctica pedagógica, es un proceso de acción y de reflexión cooperativa, de indagación y experimentación, donde el profesor aprende al enseñar, y enseña porque aprende, interviene para facilitar y no imponer sus ideas, sino más bien reflexiona sobre su intervención, lo cual permite la propia comprensión de su práctica" (p.115).
\end{abstract}

Se hace necesario que el docente abandone la enseñanza rutinaria, y se convierta en un agente de cambio para la construcción y enriquecimiento del conocimiento, apoyado en un trabajo con los estudiantes a través de situaciones de aprendizaje creativo. Al respecto Ortiz (2005) señala que en lugar de su automatismo tiene que aprender a ser un investigador, recopilar datos y analizarlos para mejorar e innovar su PP, "como el método fundamental de trabajo del docente que caracterice su estilo y modo de actuación cotidiana para llevarlo a todos los educandos" (p.55). A ello cabe agregar lo planteado por Valencia (2008), quien explica que los docentes

"nos convertimos en unos investigadores
en potencia siempre y cuando hayamos
reflexionado sobre la práctica como fuente
de conocimientos, por medio de diarios
de campo, elaboración de escritos y
comunicación de aprendizajes, porque es
así como logramos ser buenos docentes en/y
mediante la práctica pedagógica" (p.56).

De esto se infiere que la práctica sin la teoría, la investigación sin la reflexión, es ciega, pues entonces no se podría explicar y argumentar las acciones, valores, actitudes y necesidades que se generan en la interacción pedagógica. En un sentido complementario Freire (1999), indica que "la construcción del conocimiento se dará en función de la reflexión que no deberá ser una mera abstracción"; agrega que "el hombre siempre deberá ser comprendido en relación con su vínculo en el mundo" (p.69). Igualmente explica que la educación debe estar orientada hacia la acción y la reflexión de los hombres sobre la realidad, ya 
que la reflexión sin acción, se reduce al verbalismo estéril y la acción sin reflexión es activismo, porque los hombres deben actuar en el mundo para humanizarlo, transformarlo y liberarlo.

\section{Educación ambiental como disciplina pedagógica}

La Educación Ambiental (EA) como disciplina pedagógica para la transformación social, ambiental y educativa implica la evaluación, ruptura y análisis de metodologías pedagógicas, revisión de procedimientos administrativos en las instituciones y evaluación de actitudes de sus actores. Al respecto Leff (1998), plantea que

"la EA vislumbra desde el hacer, aprender haciendo; desde los conceptos cotidianos y su contraste con lo científico, lo técnico y lo revelado, la construcción de un diálogo de saberes coherente, vinculado a la realidad local, a las potencialidades y oportunidades de desarrollo de cada comunidad" (p. 245).

En este contexto, el reto de las instituciones educativas se orienta a la integración de saberes y metodologías, a la innovación educativa. Para ello la transformación y la pertinencia de la formación es esencial, dado que la dinámica del sistema educativo y los retos de la sociedad de este siglo exigen cambios en los procesos de enseñanzaaprendizaje. En un sentido formal, la EA requiere insertar sus objetivos dentro de un concepto curricular abierto, innovador y flexible, de tal manera que se logren cambios en el contexto educativo y cultural. Allí, se activa la creatividad docente, como oportunidad de investigar, donde los participantes puedan desarrollar habilidades y relacionarlas con conocimientos (García y Arana, 2000).

Estas ideas plantean, entre otras cosas, la necesidad de ubicar al individuo frente a la realidad ambiental para ir encadenando los diversos problemas en los ámbitos verdaderamente sentidos por ellos. La metodología debe enmarcarse en una visión holística e integral del ambiente, como condición necesaria en el trabajo, estudio y análisis de situaciones, fenómenos y problemas. Entonces así redescubrir el conocimiento y desarrollar una nueva cultura ambiental (Novo, 1999). En busca de tales fines la experiencia personal debe convertirse en estrategia básica, que persiga en la vivencia de procesos la observación, el estudio y análisis de situaciones de interés para los individuos.

Cabe señalar que los actuales objetivos de la EA, aun en concordancia con los declarados en Belgrado 1975, tienen que ver con sentar las bases necesarias para desarrollar actitudes positivas hacia el ambiente, despertando la capacidad de comprensión del entorno y la capacidad de evaluación crítica. Entonces se busca el 
conocimiento ambiental que active la conciencia individual y colectiva; promueva acciones donde se pongan de manifiesto valores ambientales y aptitudes en participación comprometida. También esta EA se ve desarrollada con el cumplimiento de los principios de: Totalidad, en interrelaciones sociedad-naturaleza; Permanencia a lo largo de la vida; Interdisciplinariedad, trascendiendo más allá de la disciplina; Aprendizaje activo, que supere las teorías; Identidad en los contextos que identifican al individuo y grupos sociales; Realidad actual y futura, activando acciones que cambien el presente para un futuro sostenible; Desarrollo ambiental, la complejidad ambiental trasciende a lo ecológico y social; Cooperación, como forma de alcanzar la solución de los problemas ambientales; y Glocalidad, que piensa globalmente y actúa localmente (Sauve, 2006).

\section{Naturaleza de la investigación}

La metodología fue cualitativa la cual para Casal (2007), se interesa por conocer la realidad con fines de describirla y transformarla. En tal sentido el estudio buscó conocer a profundidad la Práctica Pedagógica (PP) como fenómeno educativo complejo, capaz de ser mejorado. El método fue la investigación acción en el aula (IA), que como lo define Martínez (2006),

\footnotetext{
"es un método que por medio de la reflexión crítica y del autocuestionamiento, identifica uno o más problemas del propio desempeño docente, elabora un plan de cambio, lo ejecuta, evalúa la superación del problema y su progreso personal” (p. 244 )
}

Se asumió que la implementación de una estrategia debía partir de un diagnóstico de la situación, para luego definir claramente los objetivos que se deseaban alcanzar. Así se diseñó la estrategia, luego aplicarla y finalmente realizar la evaluación de los objetivos fijados al inicio. La IA se desarrolló en los siguientes pasos: Diagnóstico, Diseño, Ejecución y Evaluación. 


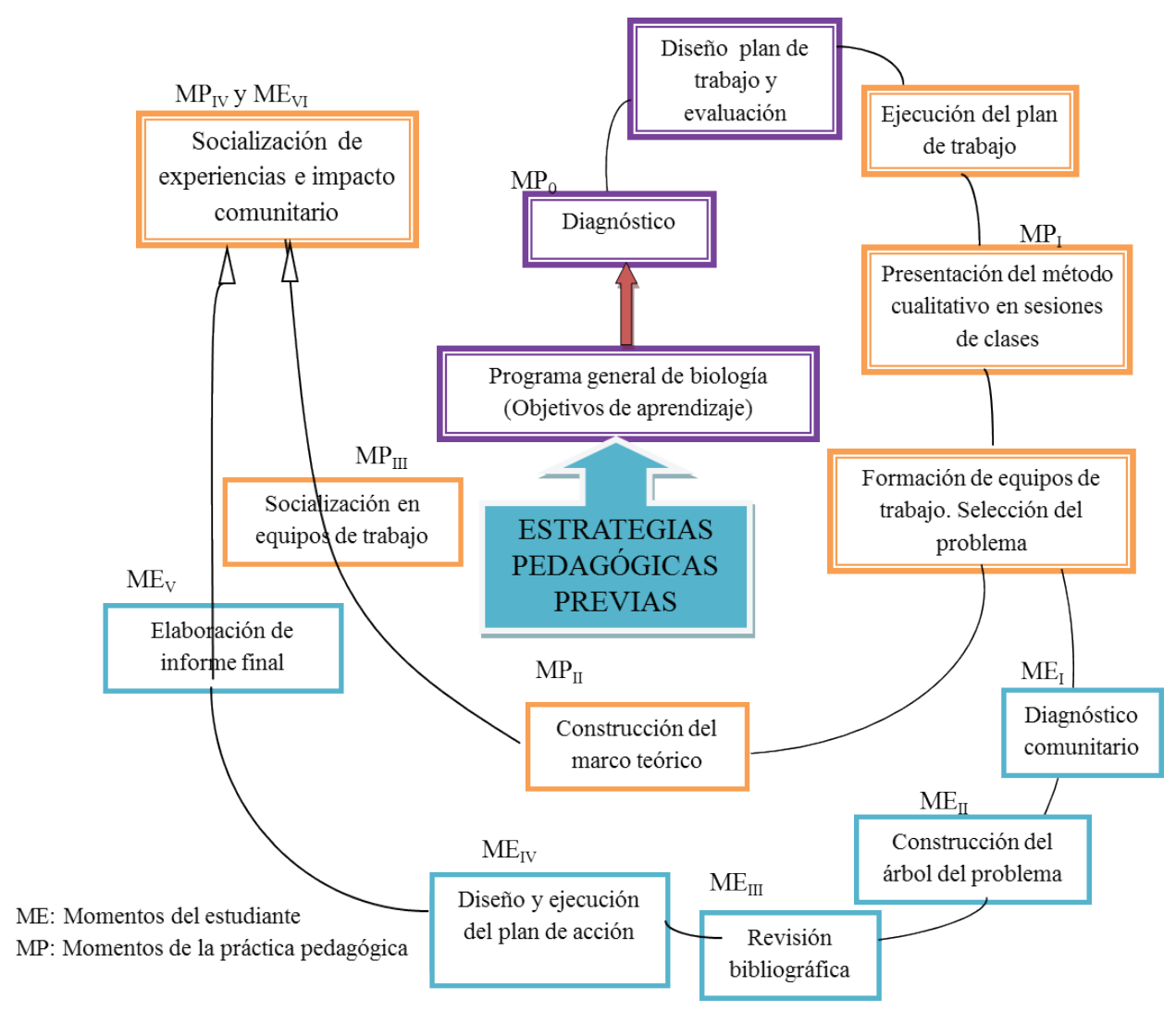

Figura 1. De momentos de la investigación acción en el aula

Todo momento fue registrado, descripción de actividades y reflexiones docentes, para luego trasladar la mayor cantidad de información posible a un texto escrito, el cual reflejara la práctica (García, 1997, p. 22). Se usaron una serie de instrumentos elaborados y otros mecanismos, que según García y Pérez (2008) ayudan "a registrar datos con gran precisión, entre ellos se pueden mencionar: pruebas fotográficas, grabaciones en video, grabaciones en audio" (p.5). Todas las narrativas expresadas en los registros fueron sistematizadas, luego categorizadas para concluir sobre la práctica pedagógica y sus impactos (Martínez, Ob.Cit. p. 265-283).

Entonces la investigación se desarrolló en diversos Momentos de la Práctica pedagógica (MP) y Momentos del Estudiante (ME), tal como se presenta en la figura 1 que se explican más adelante.

\section{Identificar conocimientos, necesidades e intereses de los estudiantes}

El diagnóstico fue parte de lo que se denominó momento "o" de la práctica pedagógica (MPo), consistió en el primer acercamiento con los estudiantes. Se partió de una lluvia de ideas realizada en cada sección, se respondieron preguntas generadoras con el fin de evidenciar sus percepciones sobre ambiente y problemáticas locales, 
así como, identificar necesidades e intereses en función de seleccionar las estrategias didácticas más apropiadas para el desarrollo del contenido programático. Para la organización de las respuestas, los estudiantes escribieron en trozos de papel sus percepciones, las cuales fueron plasmadas en un tablero por categorías, según cada pregunta generadora y haciendo uso de la frecuencia simple, se pudo cuantificar el número de respuestas que se iban repitiendo. Este ejercicio permitió presentar de forma gráfica los resultados a los estudiantes haciendo uso de estadística básica, como complemento educativo. Al respecto, cabe señalar lo planteado por Martínez (1999), donde aclara que la

"integración cualitativa-cuantitativa es válida, aunque se haya declarado un enfoque específico, sustentado en que la comprensión de fenómenos humanos puede adoptar métodos integrados con el objetivo de captar la riqueza y complejidad que estudian las ciencias sociales". (p. 79).

$51 \%$ de los estudiantes dijo que el ambiente es el conjunto de todos los seres vivos que nos rodean y su relación entre sí; mientras que el $45 \%$ dijo que el ambiente es la naturaleza y un 4\% señaló los animales como opción. Esto indica, que los estudiantes conciben principalmente el ambiente, desde una concepción naturalista, una categoría muy común en estudios de percepción como es señalado por Hidalgo (2012).

$\mathrm{Al}$ expresar los principales problemas ambientales, los estudiantes refirieron: $40 \%$ el cambio climático; $27 \%$ el agotamiento y contaminación del agua; $13 \%$ el mal manejo de los residuos sólidosy líquidos; 11\% la pérdida debiodiversidad; y $9 \%$ opinó que es la sobrepoblación. Ellos generan sus opiniones de acuerdo a la información que reciben, y en la actualidad el principal problema ambiental que se debate es el cambio climático, omitiendo otros como la pobreza. Esto amerita profundizar en una concepción más holística e integral sobre ambiente. En el mismo sentido el 68\% de los estudiantes cree que los problemas ambientales representan un peligro inminente; y el $32 \%$ opina que los problemas ambientales no tienen mucha importancia. Pareciera un cierto nivel de consciencia ambiental en los estudiantes.

Al identificar los problemas en el contexto de sus comunidades el $60 \%$ delos estudiantes percibióla existencia de contaminación ambiental; el 25\% inseguridad; el $8 \%$ embarazo en adolescentes; y un $7 \%$ otros problemas, entre los más mencionados en la encuesta están las drogas como flagelo que afecta a sus comunidades. En este sentido, se debe profundizar sobre los tipos de contaminación ambiental a los cuales hacen alusión los estudiantes. Lo importante fue que se evidenció una preocupación por el componente social de la comunidad. 
En relación al rol que juegan los estudiantes en la comunidad, seobtuvoqueel $97 \%$ creequesurolespasivoante los problemas ambientales, por tanto se requiere cambiar esta actitud, para lograr mayor interés en los estudiantes por ser parte de la solución. Esto fue en contraste con un 3\% que se sienten apáticos ante la situación de su comunidad y ningún estudiante dijo formar parte de la solución. El 56\% de los estudiantes han percibido que los habitantes de su comunidad contribuyen a los problemas existentes en la misma; un $37 \%$ opina que los habitantes de su comunidad son apáticos ante los flagelos existentes; mientras que el 7\% indica que los habitantes de su comunidad participan en la solución a los problemas. Luego el 65\% manifestó querer trabajar con proyectos de investigación para el estudio de problemáticas ambientales; el 24\% abordar la comunidad; mientras que un $11 \%$ manifestó la necesidad de trabajar en conjunto con los consejos comunales. En tal sentido, la estrategia seleccionada partió del interés y motivación de los estudiantes, reconociendo la baja participación de estudiantes y sus comunidades en solucionar los problemas ambientales existentes.

Durante éste momento de la investigación, los estudiantes mostraron interés por conocer la metodología cualitativa y abordar problemas que le eran comunes, se mostraron muy participativos. Disminuyeron las interrupciones durante la clase, se logró la atención del grupo y el proceso en el aula fue ameno. Surgieron ideas desde un punto de vista crítico sobre el abordaje de los problemas ambientales en las comunidades y se sintieron motivados a formar parte en la búsqueda de soluciones.

\section{Diseño del plan de trabajo y evaluación}

Luego del proceso reflexivo generado en el diagnóstico, se elaboró el plan de trabajo que siguió las normas de planificación del sistema de educación según el $\mathrm{CNB}, 2007$. Fue estructurado por el área de aprendizaje que correspondió al componente denominado "El ser humano y su interacción con otros componentes del ambiente" el cual tiene la finalidad de "valora la importancia de los procesos bioquímicos, transformación de la energía y procesos biotecnológicos, relacionados con la conservación y mantenimiento del equilibrio ecológico, los fenómenos, relaciones y problemas del ambiente como principio fundamental para la preservación de la vida, a partir de la comprensión del ser humano como un componente más del ecosistema. Esto como parte del componente "El ser humano con sus semejantes y otros seres vivos", dentro de la asignatura Ciencias Biológicas. Dentro de dicho componente se ubica una serie de contenidos, de los cuales se seleccionaron: cultura ambiental responsable, ejecución de actividades para la protección del ambiente y metodología de investigación. Aquí fue donde se conectó la metodología cualitativa con 
los problemas ambientales en el entorno comunitario. Se consideraron los ejes integradores: Ambiente y Salud Integral, Trabajo Liberador, Tecnología de la Información y la Comunicación.

Por su parte, el plan de evaluación se estructuró en los contenidos, según cada pilar, se definieron para lo conceptual, procedimental y actitudinal, que corresponden al conocer, hacer y convivir respectivamente; y por último fueron identintificados los indicadores para cada uno. La complejidad de los indicadores para observar el antes, durante y después del proceso, llevó a elaborar diversos instrumentos, tales como listas de verificación y escalas de estimación. También se evaluó el desarrollo de la clase, el trabajo cooperativo, la socialización de los resultados en cuanto al proceso de investigación, la memoria escrita y la presentación oral del trabajo final.

Todo el plan se desarrolló en momentos que se muestran en el esquema metodológico y se explican más adelantes según las acciones pedagógicas y los resultados.

\section{Abordaje del método científico cualitativo con equipos de estudiantes}

El momento I de la práctica pedagógica $\left(\mathrm{MP}_{\mathrm{I}}\right)$ consistió en presentar el método cualitativo a los estudiantes, facilitando una guía práctica para la elaboración de proyectos desde la metodología cualitativa. Específicamente, se explicó la investigación acción, donde se consideraron los pilares conocer y convivir. En este momento tuvieron la oportunidad de interactuar a través de mesas de trabajo, lluvias de ideas, preguntas y respuestas, durante dos sesiones de clases.

Así mismos, durante el $\mathrm{MP}_{\mathrm{I}}$ se formaron los equipos de trabajo según los intereses de los estudiantes, tratando de considerar el sector donde vivían, para facilitar el trabajo de campo. Se evidenció que en dos de las tres secciones existían problemas para integrarse y algunos de los estudiantes quedaron fuera de los grupos. En virtud de esto, se hizo necesaria la intervención docente para superar conflictos, para motivar a los estudiantes a incorporarse en los equipos ya formados. Las razones más comunes, para no incorporar ciertos estudiantes fueron la irresponsabilidad y falta de interés de algunos, según sus propios compañeros. Ante dicha dificultad se dio atención especial al grupo de estudiantes, basada en la práctica de valores como la responsabilidad, la solidaridad y el respeto, De esta manera se ejercitó el pilar convivir; como está establecido en el CNB, 2007, que supone la necesidad de formar una nueva ciudadanía con conciencia social, donde se propicien procesos colectivos, haciendo que todos se articulen por el bien común. 
Elección de temas a ser considerados por los estudiantes

Durante el mismo MP1 los estudiantes sugirieron diversas problemáticas de investigación, las cuales se organizaron en dos Categorías Medulares (CM). Una CM fue Problemas ambientales que contenía las categorías: calentamiento global, contaminación, salud integral y calidad de vida, a su vez correspondientes a 2 subcategorías Cambio climático (referidas a sequía, quemas, tala y ahorro energético) y Calidad ambiental (correspondientes a contaminación del suelo y agua, desechos sólidos y contaminación sónica). ,

La otra CM fue Problemas sociales, que correspondieron a la categoría "Salud integral y calidad de vida". Se consideraron 3 subcategorías: una Violencia y criminalidad (violencia escolar, drogas, inseguridad, pérdida de valores); dos Salud sexual y reproductiva (Embarazo en adolescentes, aborto, ETS); y tres fue Trastornos nutricionales (anorexia y bulimia).

Se formaron entre las tres secciones quince (15) equipos, lo que corresponde a cinco (5) equipos por sección, cada uno de acuerdo a su interés y motivación seleccionaron el problema ambiental y la comunidad a abordar; en total fueron 11 barriadas guanareñas. En ese sentido, el pilar conocer se observó durante éste proceso, ya que como lo dice Delors (1996), consiste en aprender a comprender el mundo que le rodea, conocer y descubrir (p. 3). Aquí, los estudiantes lograron incrementar el saber, comprendieron mejor sus entornos. Igualmente se favoreció la curiosidad intelectual, se estimuló el sentido crítico y se permitió descifrar la realidad, adquiriendo al mismo tiempo una autonomía de juicio.

\section{Trabajo de campo del estudiante}

El momento I del estudiante $\left(\mathrm{ME}_{\mathrm{I}}\right)$, consistió en un primer acercamiento a la comunidad con el fin de realizar el diagnóstico, recopilando toda la información necesaria a través de la observación. Los estudiantes construyeron dentro del aula, el instrumento de recolección de información, el cual fue aplicado en cada comunidad seleccionada. En éste paso, el equipo realizó su primer contacto con los miembros de la comunidad con la finalidad de incentivarlos y sensibilizarlos en cuanto a la necesidad de participar. Así cada equipo logró la elaboración del diagnóstico, con lo cual se determinó el o los problemas que demandan solución.

Éste primer acercamiento fue muy significativo para los estudiantes, provocando una actitud investigativa, tuvieron la oportunidad de recorrer su propia comunidad desde una perspectiva distinta. Pusieron en práctica la observación, miraron problemas que hasta ese 
momento eran ajenos para ellos. En este paso, los estudiantes aplicaron una entrevista a los habitantes de la comunidad para diagnosticar cómo percibían los mismos las problemáticas ambientales que emergieron.

\section{Árbol del problema, definición de objetivos}

Luego de la observación de la realidad ambiental comunitaria siguió el momento II del estudiante $\left(\mathrm{ME}_{\mathrm{II}}\right)$, donde se realizó el árbol del problema en el aula de clases. En él expresaron el encadenamiento tipo causa/efecto, de las condiciones negativas percibidas en relación con el problema en cuestión. De allí que, los estudiantes/ investigadores por sí solos pudieron construir el objetivo general y los objetivos específicos que serviría de guía en todo el proceso.

Los logros durante este momento se resumen de la siguiente manera: Reflexión por parte de los estudiantes sobre causas y efectos de los problema seleccionado, viendo el ambiente con una visión holística e integrada; Concientización en la responsabilidad personal sobre los problemas ambientales; Fortalecimiento del trabajo cooperativo; Se produjo la generación de ideas espontáneas; y Se logró la contextualización del aprendizaje al analizar los diversos problemas ambientales comunitarios.

Revisión bibliográfica y construcción del marco teórico

El momento III del estudiante $\left(\mathrm{ME}_{\mathrm{III}}\right)$, fue donde buscaron toda la información sobre el tema, que sirviera de base teórica para el estudio del problema. Durante éste momento los estudiantes tuvieron la oportunidad de realizar la revisión de las fuentes necesarias. Indagaron sobre conceptos, teorías, principios, características, causas, consecuencias, entre otros; así como también, otras investigaciones asociadas con el tema seleccionado y el cúmulo de leyes que pudieran servir de base en la investigación.

Este evento generó un profundo proceso reflexivo en la práctica pedagógica, el cual se abordó durante la construcción del marco teórico en el momento II de la misma $\left(\mathrm{MP}_{\mathrm{II}}\right)$. La contextualización de los contenidos teóricos en el abordaje del problema comunitario, permitió la construcción del conocimiento por parte de los estudiantes, alcanzando un aprendizaje significativo.

\section{Enfrentar el problema ambiental}

El momento IV del estudiante $\left(\mathrm{ME}_{\mathrm{IV}}\right)$, fue donde diseñaron y ejecutaron un plan de acción dirigido a la búsqueda de soluciones de los problemas ambientales 
detectados; evidenciándose significativamente los indicadores del pilar hacer. Los estudiantes definieron el objetivo general, los objetivos específicos, las actividades, las estrategias, los recursos necesarios, los responsables, el lapso de ejecución y evaluación de los resultados. En este punto, los estudiantes, establecieron prioridades ante las necesidades, y se plantearon opciones entre las posibles alternativas.

Ahora bien, enfrentar la acción supone poner en práctica la planificación y la observación de sus efectos en el contexto en el que tiene lugar la investigación. Aquí es importante el trabajo en equipo para llevar a cabo las actividades diseñadas. De igual modo, esto exige un juicio crítico sobre los procesos, problemas y restricciones que se manifiestan y sobre sus efectos. Esta acción, ayuda a valorarla desde lo previsto y deseable, la posibilidad de sugerir un nuevo plan, si es necesario.

Ya ejecutado el plan, los estudiantes elaboraron el informe final $\left(\mathrm{ME}_{\mathrm{v}}\right)$, donde pudieron comunicar por escrito el resultado de la investigación, haciendo uso de las normas solicitadas y el eje transversal Tecnología de la Información y Comunicación. Este exigió que el equipo de trabajo organice toda la información y la estructurará en base a los pasos del esquema proporcionado. Hicieron esfuerzos por dar coherencia, cohesión y corrección lingüística y estilística, de acuerdo con los criterios formales de presentación de trabajos.

\section{Socialización de la experiencia docente-estudiantes}

La socialización se dio en un continuo de encuentros destacando dos momentos de la práctica pedagógica el III $\left(\mathrm{MP}_{\text {III }}\right)$, se llevó a cabo en equipos de trabajos, donde a través de una discusión colectivizada, se detectaban los logros personales y grupales, igualmente se solventaban dificultades.

Luego el momento IV de la práctica pedagógica $\left(\mathrm{MP}_{\mathrm{IV}}\right)$ y momento VI del estudiante $\left(\mathrm{ME}_{\mathrm{VI}}\right)$ fue la finalización de la ejecución del plan de trabajo por parte de los estudiantes. Aquí se propició un ambiente de participación, donde los integrantes de cada equipo expresaron la experiencia de su investigación y se valoró el impacto comunitario de la misma. Esto correspondió a momentos importantes de la evaluación donde los estudiantes realizaron la reflexión crítica sobre el aprendizaje alcanzado, el reforzamiento de valores como la responsabilidad, el respeto, el trabajo en equipo, entre otros.

Durante esta actividad, los estudiantes presentaron de forma oral y escrita, los resultados obtenidos según las fases de su investigación. Se realizó una exposición pública de cada equipo de trabajo, donde con más rigurosidad explicaron el proceso y los logros alcanzados. Éste ejercicio, permitió, tanto para los estudiantes como para la docente: Identificar los logros personales y grupales; Fomentar la 
participación; Reflexionar de manera crítica y constructiva ante situaciones de aprendizaje; Opinar sobre la actuación personal dentro del equipo y la integración entre los miembros; Mejorar la responsabilidad e identificación con el trabajo y emitir juicios valorativos acerca de otros en un entorno de libertad, compromiso y responsabilidad.

\section{Evaluación}

Por ser una investigación acción en aula (IA), la evaluación fue un proceso continuo durante el ejercicio pedagógico. La misma sellevó a cabo desde dos perspectivas: por un lado evaluando el impacto de la Práctica Pedagógica (PP) en el abordaje de problemas ambientales en las comunidades seleccionadas, y por la otra evaluando la propia PP. Aquí la observación del proceso fue esencial, así como señala Álvarez (2003) que la misma es "la piedra angular del conocimiento, la cual ofrece la ventaja de una mayor profundidad e introspecciones en los significados y experiencias" (p. 81). A lo largo de la investigación, el docente reflexionaba y repensaba su forma de proceder en función de los resultados esperados y alcanzados, basado en la actitud de los estudiantes, la motivación alcanzada, dificultades presentadas y la profundidad conceptual desarrollada.

Este espacio del artículo se presenta en los dos sentidos expuestos, uno se relaciona con los impactos comunitarios de la PP y el otro con la evaluación de la PP, siendo este la esencia de la investigación.

\section{Impactos comunitarios de la práctica pedagógica}

Se lograron desarrollar 15 proyectos con impacto en 11 barrios, los temas más recurrentes fueron: Uso racional de energía eléctrica, Contaminación de agua en canales; Manejo inadecuado de residuos sólidos en áreas públicas. La práctica pedagógica permitió en la comunidad: Identificar y abordar problemas ambientales en el contexto comunitario de los estudiantes; Pensar y activar soluciones a problemas; Despertar interés en los estudiantes y comunidades sobre los problemas ambientales de sus contextos locales; y Fortalecer la relación escuela-familia-comunidad.

Las acciones se orientaron a: Concientización ambiental de las comunidades a través de conversatorios y talleres; Evaluación delas condiciones ambientales de áreas públicas; y Restauración de espacios públicos.

\section{Evaluación de la práctica pedagógica}

La valoración de la Práctica Pedagógica (PP) se dio a todo lo largo de su desarrollo, tal como se ha venido 
expresando. Durante la PP fue registrado y evaluado, como contante para el proceso de reflexión y acción. Cada momento fue evaluado según los indicadores que fueron definidos en el diseño de la PP, que se muestran en la Tabla 1.

Ahora bien, se asumieron tres aspectos fundamentales enla evaluación: laopinión sobreel proceso deinvestigación; la percepción sobre la importancia de la aplicación del proyecto en el nivel académico que fue realizado; y la percepción sobre la importancia de la metodología cualitativa para abordar problemas ambientales en las comunidades. En tal sentido, a continuación se presenta un cuadro donde se muestran los indicadores según el pilar conocer, hacer y convivir; en otra columna se señalan las técnicas aplicadas y los instrumentos utilizados.

Tabla 1. Indicadores de evaluación definidos en el diseño de la práctica pedagógica

\begin{tabular}{|c|c|c|c|c|}
\hline & $\begin{array}{c}\text { Pilares } \\
\text { Indicadores }\end{array}$ & & \multirow[t]{2}{*}{ Técnicas } & \multirow[t]{2}{*}{ Instrumentos } \\
\hline Conocer & Hacer & Convivir & & \\
\hline $\begin{array}{c}\text { Aplica los pasos de la } \\
\text { metodología cualitativa }\end{array}$ & $\begin{array}{l}\text { Selecciona y plantea } \\
\text { el problema de } \\
\text { investigación }\end{array}$ & $\begin{array}{c}\text { Práctica valores } \\
\text { individuales, sociales } \\
\text { y universales }\end{array}$ & \multirow{5}{*}{$\begin{array}{c}\text { Observación } \\
\text { Mesas de trabajo } \\
\text { Lluvia de ideas } \\
\text { Preguntas y respuestas } \\
\text { Trabajo de campo } \\
\text { Reuniones de } \\
\text { acompañamiento } \\
\text { Trabajo escrito } \\
\text { Exposición }\end{array}$} & \multirow{5}{*}{$\begin{array}{c}\text { Lista de verificación } \\
\text { (cotejo) }\end{array}$} \\
\hline $\begin{array}{l}\text { Describe y analiza el } \\
\text { problema seleccionado }\end{array}$ & $\begin{array}{c}\text { Tiene coherencia en } \\
\text { las ideas }\end{array}$ & $\begin{array}{c}\text { Muestra interés por el } \\
\text { problema }\end{array}$ & & \\
\hline Genera soluciones a las & & & & \\
\hline problemáticas ambientales & $\begin{array}{l}\text { Expresa con claridad } \\
\text { la metodología }\end{array}$ & Participación activa & & \\
\hline Emite opiniones críticas & aplicada & Apoyo mutuo & & \\
\hline \multirow{2}{*}{$\begin{array}{l}\text { Contextualiza el } \\
\text { aprendizaje }\end{array}$} & Registra los avances & Perseverancia & & \\
\hline & $\begin{array}{c}\text { Escribe según las } \\
\text { normas entregadas }\end{array}$ & $\begin{array}{l}\text { Socializa la } \\
\text { experiencia }\end{array}$ & & \\
\hline
\end{tabular}

\section{Opinión de los estudiantes sobre el proceso de investigación}

Resumiendo las categorías emergidas de las opiniones de los estudiantes sobre el proceso de investigación, el mismo representó: Una experiencia significativa, Trabajo en equipo, Crecimiento personal, Demostración de destrezas, Colaboración, Formación académica, Obtención de resultados, Complejidad significativa, y Agentes promotores de conciencia ambiental. Todos se presentan en la Tabla 2, las categorías descriptivas fueron cruzadas con los principios y objetivos de la EA y los pilares de la educación de la educación bolivariana (conocer, hacer, convivir). En tal sentido, se buscó dar validación desde las exigencias del marco educativo y la educativa ambiental.

En la Tabla 2 se puede leer, entre otras cosas, que la investigación dentro de las comunidades representó una experiencia de aprendizaje significativo. Fue una oportunidad de trabajo en equipo, crecimiento personal 
y una posibilidad de demostrar destrezas y colaboración. En tal sentido la PP evidenció la posibilidad de cumplir con el principio de cooperación de la EA y capacidad de resolución de problemas; así como los objetivos de actitudes y aptitudes favorables para asumir situaciones ambientales de sus comunidades, reflejando con ello la posibilidad de aplicar el pilar de convivir establecido en el sistema educativo.

Tabla 2. Categorías emergidas de las opiniones de los estudiantes sobre el proceso de investigación

\begin{tabular}{ccccc}
\hline \multirow{2}{*}{$\begin{array}{c}\text { Unidad } \\
\text { Temática }\end{array}$} & Categorías descriptivas & \multicolumn{2}{c}{ Evidencia de los } \\
\cline { 2 - 5 } & $\begin{array}{c}\text { Experiencia significativa, trabajo } \\
\text { en equipo, crecimiento personal, } \\
\text { demostración de destrezas, } \\
\text { colaboración }\end{array}$ & $\begin{array}{c}\text { Principios } \\
\text { de la EA }\end{array}$ & $\begin{array}{c}\text { Objetivos } \\
\text { de la EA Eacidad de resolución de }\end{array}$ & Pilares \\
\hline problemas & Actitud & Aptitud & Convivir \\
\cline { 2 - 5 } $\begin{array}{c}\text { Valoración de } \\
\text { la experiencia: } \\
\text { opinión de los } \\
\text { estudiantes }\end{array}$ & $\begin{array}{c}\text { Formación académica, obtención de } \\
\text { resultados, complejidad significativa }\end{array}$ & $\begin{array}{c}\text { Interdisciplinariedad } \\
\text { Aprendizaje activo }\end{array}$ & Conocimiento & Conocer \\
\cline { 2 - 5 } & $\begin{array}{c}\text { Agentes promotores de conciencia } \\
\text { ambiental, }\end{array}$ & $\begin{array}{c}\text { Desarrollo ambiental } \\
\text { Cooperación } \\
\text { Glocalidad }\end{array}$ & Participación & Conocer \\
& $\begin{array}{c}\text { Investigación dentro de las } \\
\text { comunidades. }\end{array}$ & Conciencia & Hacer \\
\hline
\end{tabular}

\section{Percepción estudiantil sobre la importancia}

\section{académica de la aplicación de la PP}

Resumiendo las categorías emergidas de las opiniones de los estudiantes, sobre la importancia académica de la aplicación de la PP, las mismas fueron: La investigación como técnica de estudio; Conocimiento práctico; Descubrir conocimientos que le serán de utilidad en niveles superiores; y Contextualización de la enseñanza. Todos se presentan en la tabla 3 y se relacionan con los principios y objetivos de la EA y los pilares educativos.

Tabla 3. Categorías emergidas de las opiniones de los estudiantes, sobre la importancia académica de la aplicación de la PP

\begin{tabular}{|c|c|c|c|c|}
\hline \multirow{2}{*}{$\begin{array}{l}\text { Unidad } \\
\text { Temática }\end{array}$} & \multirow[b]{2}{*}{ Categorías descriptivas } & \multicolumn{3}{|c|}{ Evidencia de los } \\
\hline & & $\begin{array}{c}\text { Principios } \\
\text { de la EA }\end{array}$ & $\begin{array}{c}\text { Objetivos } \\
\text { de la EA }\end{array}$ & Pilares \\
\hline \multirow{8}{*}{$\begin{array}{l}\text { Importancia de } \\
\text { la aplicación del } \\
\text { proyecto en éste } \\
\text { nivel académico }\end{array}$} & \multirow{2}{*}{$\begin{array}{l}\text { La investigación como } \\
\text { técnica de estudio, conocimiento } \\
\text { práctico y útil. }\end{array}$} & \multirow[b]{2}{*}{ Aprendizaje activo } & Conocimiento & Conocer \\
\hline & & & $\begin{array}{l}\text { Capacidad de } \\
\text { evaluación }\end{array}$ & Hacer \\
\hline & $\begin{array}{l}\text { Descubrir conocimientos que } \\
\text { le serán de utilidad en niveles } \\
\text { superiores }\end{array}$ & $\begin{array}{l}\text { Permanencia } \\
\text { Realidad actual y } \\
\text { futura }\end{array}$ & Conocimiento & Conocer \\
\hline & \multirow{5}{*}{$\begin{array}{l}\text { Contextualización de la enseñanza: } \\
\text { Conciencia de la realidad ambiental, } \\
\text { socialización comunitaria y calidad } \\
\text { de vida }\end{array}$} & \multirow{5}{*}{$\begin{array}{l}\text { Realidad actual y } \\
\text { futura } \\
\text { Interdisciplinariedad } \\
\text { Glocalidad } \\
\text { Cooperación }\end{array}$} & conciencia & \multirow{5}{*}{$\begin{array}{l}\text { conocer } \\
\text { Hacer } \\
\text { Convivir }\end{array}$} \\
\hline & & & Actitud & \\
\hline & & & & \\
\hline & & & Aptitud & \\
\hline & & & Participación & \\
\hline
\end{tabular}


En la Tabla 3 se puede leer, entre otras cosas, que para los estudiantes la investigación representó una técnica de estudio, que permitió conocimiento práctico y útil. En tal sentido se evidenció la posibilidad de cumplir en la PP con el principio de aprendizaje activo de la EA y con los objetivos de conocimiento y capacidad de evaluación, reflejando con ello la posibilidad de aplicar el pilar de conocer y hacer establecido en el sistema educativo.

\section{Percepción estudiantil sobre la importancia de la metodología cualitativa}

Resumiendo las categorías emergidas de las opiniones de los estudiantes, sobre la Importancia de la investigación cualitativa, las mismas fueron: Permite percibir los problemas ambientales, a través del contacto directo con la comunidad; Conciencia ambiental; Solución de problemas; y Corresponsabilidad ambiental. Todos se presentan en la Tabla 4 y se relacionan con los principios y objeticos de la EA y los pilares educativos.

Tabla 4. Categorías emergidas de las opiniones de los estudiantes, sobre la importancia de la investigación cualitativa

\begin{tabular}{|c|c|c|c|c|}
\hline \multirow[b]{2}{*}{$\begin{array}{l}\text { Unidad } \\
\text { temática }\end{array}$} & \multirow[b]{2}{*}{ Categorías descriptivas } & \multicolumn{3}{|c|}{ Evidencia de los } \\
\hline & & $\begin{array}{l}\text { Principios } \\
\text { de la EA }\end{array}$ & $\begin{array}{c}\text { Objetivos } \\
\text { de la EA }\end{array}$ & Pilares \\
\hline \multirow{5}{*}{$\begin{array}{l}\text { Importancia de } \\
\text { la investigación } \\
\text { cualitativa para } \\
\text { abordar problemas } \\
\text { ambientales en las } \\
\text { comunidades }\end{array}$} & $\begin{array}{l}\text { Permite percibir los problemas } \\
\text { ambientales, a través del contacto } \\
\text { directo con la comunidad }\end{array}$ & $\begin{array}{c}\text { Totalidad } \\
\text { Realidad actual y } \\
\text { futura } \\
\text { Glocalidad }\end{array}$ & $\begin{array}{c}\text { Participación } \\
\text { Capacidad de } \\
\text { evaluación }\end{array}$ & $\begin{array}{l}\text { Conocer } \\
\text { Convivir }\end{array}$ \\
\hline & Conciencia ambiental & $\begin{array}{c}\text { Totalidad } \\
\text { Identidad nacional }\end{array}$ & $\begin{array}{c}\text { Actitud } \\
\text { Conciencia } \\
\text { Participación }\end{array}$ & $\begin{array}{c}\text { Conocer } \\
\text { Hacer }\end{array}$ \\
\hline & & & & Conocer \\
\hline & Resolución de problemas & $\begin{array}{l}\text { Cooperación } \\
\text { Desarrollo ambiental } \\
\text { Glocalidad }\end{array}$ & $\begin{array}{c}\text { Aptitud } \\
\text { Participación }\end{array}$ & Hacer \\
\hline & & & & convivir \\
\hline
\end{tabular}

En la Tabla 4 se puede leer, entre otras cosas, que la investigación cualitativa para los estudiantes representó una oportunidad para percibir los problemas ambientales, a través del contacto directo con la comunidad. En tal sentido se evidenció la posibilidad de cumplir en la PP con el principio de educación ambiental a través de la una visión de Totalidad e integralidad de la realidad ambiental, Realidad actual y futura y Glocalidad en la forma de pensar globalmente en los problemas pero actual localmente. También permitió cumplir con los objetivos de conocimiento y capacidad de evaluación de situaciones ambientales, reflejando con ello la posibilidad de aplicar el pilar de conocer y convivir establecido en el sistema educativo bolivariano. 


\section{Consideraciones finales}

La práctica pedagógica (PP) que considera la enseñanza de la biología y metodología cualitativa en el contexto de vida de los estudiantes, permite incorporar de manera transversal la dimensión ambiental y la investigación como partes de la formación integral. Este escenario permite que la teoría y la práctica adquieran sentido, al inscribirse en la realidad comunitaria de los estudiantes, motivando la creatividad en el hacer con consciencia. El enfoque holístico e integrador posibilita la construcción de un pensamiento crítico en el acercamiento al conocimiento y realidad ambiental. Con la PP se logró fomenta el trabajo en equipo y la construcción colaborativa, en una práctica constante de valores del ser y convivir.

La contextualización del aprendizaje con problemas ambientales en sectores locales, despierta el interés del estudiante, y ayuda a fortalecer la relación comunidad-escuela para el abordaje de problemas ambientales en el contexto local. Esto permite que el estudiante valore la realidad ambiental y se relacione con ella, pase de una condición de indiferencia a protagonismo consciente.

La PP ayuda a superar la enseñanza disciplinar, logrando que el estudiante al querer profundizar, para el entendimiento de cada tema ambiental, recurriera al conocimiento de otras disciplinas. En tal sentido, conduce a la interdisciplinariedad, a la transdisciplinariedad a través de métodos activos y participativos; convirtiendo al educando en agentes de su propio aprendizaje. Existe con esto la posibilidad del cambio de papel del docente, de poseer y transmitir el conocimiento pasa a gestionar el aprendizaje del estudiante, lo que en la actualidad se le ha denominado aprender a aprender.

Cuando el docente es reflexivo y crítico de su hacer educativo, puede convertirse en un constante investigador de su PP lo que le permite estar en una innovación constante para mejor la calidad de la educación. Ese investigador, que es el mismo docente, se apoya de la constante observación, reflexión y acción para el mejoramiento de la PP. Igualmente en ese proceso logra superar dificultades durante la PP asumiendo una actitud flexible y activa para resolver conflictos que se presenten.

El docente educador ambiental, independientemente cual sea la disciplina como parte de exigencias curriculares, puede trabajar los temas ambientales desde un enfoque crítico con los estudiantes, que logre sensibilizarlos y transformarlos en seres activos y responsables ante la realidad ambiental. Puede a través de la PP cumplir con los objetivos y principios de la educación ambiental, propiciando un aprendizaje activo, sustentado en el conocimiento ambiental, propiciando actitudes y aptitudes para solventar problemas ambientales desde la participación en la realidad. 


\section{Referencias}

Álvarez, L. 2003. Cómo hacer investigación cualitativa: Fundamentos y metodología. México, D.F: Paidós; p. 81

Casal, 2007. Marco Metodológico. Como hacer una propuesta de investigación. [Documento en línea]. En https://docs. google.com/viewer. [Consulta: Junio 15, 2017]; p.15

Delors, J. 1996. La educación encierra un tesoro. Informe a la UNESCO de la Comisión Internacional sobre la educación para el siglo XXI. Madrid: Santillana. Ediciones UNESCO. En: http://www.oei.es/decada/accion.php?accion=004. [consulta febrero 18 de 2013].

Freire, P. 1999. Pedagogía del Oprimido, Editorial Siglo Veintiuno, México. P.69

García, H. y Pérez, J. 2008. La investigación del profesor en el aula. [Documento en línea]. En: https://html. rincondelvago.com/investigacion-en-el-aula.html [Consulta: Marzo 22, 2017], p.5.

García, A. 1997. La instrumentación metodológica en la recuperación de la práctica docente. Unidad editorial del Gobierno del Estado de Jalisco.

García, M. y Arana, A. 200o. Origen, metas, objetivos, principios y paradigmas de la Educación ambiental. FEDUPEL. Caracas. P. 20-21. México. p. 22.

Hidalgo, C. 2012. Transformación de la praxis educativa ambiental de docentes en la Universidad Centroccidental Lisandro Alvarado. Tesis de Doctorado en educación Ambiental, Universidad Pedagógica Libertador, Caracas.

Leff, E. 1998. El saber ambiental: sustentabilidad, racionalidad, complejidad y poder. México: Siglo XXI PNUMA; p.245.

Martínez, M. 2006. La investigación cualitativa. Síntesis conceptual. Revista IIPS. V $9 \mathrm{n}^{\circ} 1$; p.132-146, 244, 265-283.

Martínez, M. (1999). Criterios para la Superación del Debate Metodológico "Cuantitativo/Cualitativo". Revista Interamericana de Psicología. 1 (33), 79-107.

Martínez, N. 2006. La práctica docente motivo de reflexión. Congreso Estatal de Investigación Educativa. Actualidad, Prospectivas y Retos. http://portalsej.jalisco.gob.mx/ investigacion-.pdf; p. 34 .

Novo, M. 1999. La educación ambiental. Bases éticas, conceptuales y metodológicas. Madrid, España: Editorial Universitas. p. 122-124 
Ortiz, O. 2005. Centro de Estudios Pedagógicos y didácticos CEPEDID. Barranquilla Colombia. p.55.

Rodríguez, G. 2006. Práctica pedagógica. Una tensión entre la realidad y la práctica. En: Pedagogía y saberes. $\mathrm{N}^{\mathrm{o}}$ 24. P. 115 .

Sauvé, L. 2006. La Educación Ambiental Y La Globalización: Desafíos Curriculares Y Pedagógicos. Revista Iberoamericana De Educación. En: http://www. oei.es/oeivirt/educambien.htm. [consulta mayo 10 2017]. N. ${ }^{\circ} 41$, pp. 83-101

Valencia, S. 2008. La práctica pedagógica: un espacio de reflexión. Universidad de Antioquia, Instituto Universitario de Educación Física. Medellín, Colombia. [Documento en línea] en: http://viref.udea.edu.co/contenido/practic. P.7

Venezuela. Currículo Nacional Bolivariano. CNB 2007. Ministerio del Poder Popular para la Educación.

Recebido em: 20/07/2019

Aprovado em: 22/10/2019

Publicado em: 20/12/2019 